\title{
SOME ALGEBRAICALLY INDEPENDENT CONTINUED FRACTIONS
}

\author{
VICHIAN LAOHAKOSOL AND PATCHARA UBOLSRI
}

\begin{abstract}
Using simple arguments, we prove algebraic independence of a class of continued fractions extending an earlier result of Bundschuh. We then apply it to give another proof of algebraic independence of numbers whose $g$-adic and continued fraction expansions are explicitly known.
\end{abstract}

1. Introduction. Let

$$
A=a_{0}+\frac{1}{a_{1}+} \frac{1}{a_{2}+} \frac{1}{a_{3}+\ldots}=\left[a_{0} ; a_{1}, a_{2}, a_{3}, \ldots\right]
$$

and

$$
B=b_{0}+\frac{1}{b_{1}+} \frac{1}{b_{2}+} \frac{1}{b_{3}+\ldots}=\left[b_{0} ; b_{1}, b_{2}, b_{3}, \ldots\right]
$$

be continued fractions with positive integral partial quotients. Recently, Bundschuh $[3]$ proved that if there exists a real number $r>1$ such that

$$
r^{-1} a_{n} \geq b_{n} \geq a_{n-1}^{n-1} \quad(n=1,2,3, \ldots),
$$

then $A$ and $B$ are algebraically independent (over $\mathbf{Q}$ ). Bundschuh's proof makes use of a criterion for algebraic independence established by Durand [4]. In this note, we shall prove this same theorem under a weaker hypothesis. Our method does not appeal to Durand's criterion, but is based on an algebraic independence proof of Shiokawa [7] for gap series. Shiokawa mentioned that his arguments were essentially due to Flicker [5]. We now formulate our main result.

THEOREM. Let $A, B,\left(a_{n}\right),\left(b_{n}\right)$ be as above. Let $r>1,\left(n_{j}\right)$ be an increasing sequence of positive integers and let $f(n)$ be an integer-valued function of natural argument $n$ with $f(n) \geq 2(n=0,1,2, \ldots)$, and $f\left(r_{j}\right) \rightarrow \infty(j \rightarrow \infty)$. If

$$
r^{-1} a_{n} \geq b_{n} \geq a_{n-1}^{f(n-1)} \quad(n=1,2,3, \ldots),
$$

then $A$ and $B$ are algebraically independent (over $\mathbf{Q}$ ).

As an application, we shall prove

COROLlaRY. Let $\beta$ be a positive irrational number, $g_{1} \geq g_{2}$ be two distinct natural numbers $>1$. If the simple continued fraction of $\beta$ has unbounded partial quotients each of which is $\geq 1+\left(2 \log g_{1}\right) / \log g_{2}$, then the two numbers

$$
\sum_{i=1}^{\infty}\left(g_{t}-1\right) g_{t}^{-[i \beta]} \quad(t=1,2),
$$

where $[x]$ denotes the integer part of $x$, are algebraically independent (over $\mathbf{Q}$ ).

Received by the editors September 21, 1984.

1980 Mathematics Subject Classification. Primary 10F37, 10F20.

Key words and phrases. Algebraic independence, continued fractions, $g$-adic expansion. 
The two algebraically independent numbers in the corollary are interesting because their $g$-adic developments (as defined above) and their continued fractions can explicitly be computed. They have recently become better known owing to the works of Adams and Davison [1] and Bundschuh [2]. Their algebraic independence, under a slightly weaker hypothesis on the partial quotients, was first proved by Bundschuh [2] using Durand's criterion.

2. Lemma. We shall first recall an auxiliary result. For $A, B,\left(a_{n}\right),\left(b_{n}\right)$ as above, let the $n$th convergents of $A, B$ be, respectively,

$$
\frac{p_{n}(A)}{q_{n}(A)}=\left[a_{0} ; a_{1}, \ldots, a_{n}\right], \quad \frac{p_{n}(B)}{q_{n}(B)}=\left[b_{0} ; b_{1}, \ldots, b_{n}\right] \quad(n=0,1, \ldots) .
$$

LEMMA. Let the notation be as above. Then

(i)

$$
\begin{aligned}
0 & <\left|A-p_{n}(A) / q_{n}(A)\right| \leq\left(q_{n}(A) q_{n+1}(A)\right)^{-1} \leq\left(a_{n+1} q_{n}^{2}(A)\right)^{-1}, \\
0 & <\left(2 q_{n}(B) q_{n+1}(B)\right)^{-1} \leq\left|B-p_{n}(B) / q_{n}(B)\right| \\
& \leq\left(q_{n}(B) q_{n+1}(B)\right)^{-1} \leq\left(b_{n+1} q_{n}^{2}(B)\right)^{-1} .
\end{aligned}
$$

(ii) If $a_{n} \geq r b_{n}(r>1)$ for all $n=1,2, \ldots$, then

$$
q_{n}(A) \geq r^{n / 2} q_{n}(B) \quad(n=0,1,2, \ldots) .
$$

(iii) If $\left(a_{n}\right)$ is a strictly increasing sequence and $a_{n+1} \geq a_{n}^{2}(n=2,3,4, \cdots)$, then

$$
q_{n}(A) \leq a_{n}^{2} \quad(n=0,1,2, \ldots) .
$$

Proof. (i) is standard for any continued fractions; see e.g. Chapter X of Hardy and Wright [6].

(ii) is Lemma 3 of Bundschuh [3]; it follows easily from induction.

(iii) is Lemma 1(iv) of Bundschuh [3]; again the proof is easily done by induction.

3. Proof of the Theorem. Suppose on the contrary that $A$ and $B$ are algebraically dependent (over $\mathbf{Q}$ ). Then there exits a nonidentically vanishing

$$
P(X, Y):=\sum_{i=0}^{D_{1}} \sum_{j=0}^{D_{2}} w_{i j} X^{i} Y^{j} \in \mathbf{Z}[X, Y]
$$

such that $P(A, B)=0$. We may assume that $P(X, Y)$ is one with minimum total degree $D_{1}+D_{2}$ among such polynomials. Consider for fixed natural number $n$

$$
P_{n}:=P\left(\frac{p_{n}(A)}{q_{n}(A)}, \frac{p_{n}(B)}{q_{n}(B)}\right)=\sum_{i, j} w_{i j}\left(\frac{p_{n}(A)}{q_{n}(A)}\right)^{i}\left(\frac{p_{n}(B)}{q_{n}(B)}\right)^{j} .
$$

If $P_{n} \neq 0$, then being a rational number we must have

$$
\left|P_{n}\right| \geq\left(q_{n}(A)\right)^{-D_{1}}\left(q_{n}(B)\right)^{-D_{2}}
$$

and so, using part (ii) of the lemma,

$$
\left|P_{n}\right| \geq r^{n D_{2} / 2}\left(q_{n}(A)\right)^{-D_{1}-D_{2}}
$$


Let

$$
\begin{aligned}
& \delta_{1}:=\delta_{1}(n, A)=A-p_{n}(A) / q_{n}(A), \\
& \delta_{2}:=\delta_{2}(n, B)=B-p_{n}(B) / q_{n}(B) .
\end{aligned}
$$

Then

$$
\begin{aligned}
P_{n} & =P\left(A-\delta_{1}, B-\delta_{2}\right)=\sum_{i, j} w_{i j}\left(A-\delta_{1}\right)^{i}\left(B-\delta_{2}\right)^{j} \\
& =w_{1} \delta_{1}+w_{2} \delta_{2}+O\left(|\delta|^{2}\right)
\end{aligned}
$$

where $|\delta|=\max \left(\left|\delta_{1}\right|,\left|\delta_{2}\right|\right)$,

$$
w_{1}=-\sum_{i, j} i w_{i j} A^{i-1} B^{j}, \quad \text { and } \quad w_{2}=-\sum_{i, j} j w_{i j} A^{i} B^{j-1} .
$$

By part (i) of the lemma and by the hypotheses of the theorem, we get

$$
\left|\delta_{1}\right|=\left|A-p_{n}(A) / q_{n}(A)\right| \leq\left(a_{n+1} q_{n}^{2}(A)\right)^{-1} \leq\left(r a_{n}^{f(n)} q_{n}^{2}(A)\right)^{-1},
$$

and so by part (iii) of the lemma,

$$
\left|\delta_{1}\right| \leq r^{-1}\left(q_{n}(A)\right)^{-2-f(n) / 2} .
$$

Similarly,

$$
\begin{aligned}
\left|\delta_{2}\right| & =\left|B-p_{n}(B) / q_{n}(B)\right| \leq\left(b_{n+1} q_{n}^{2}(B)\right)^{-1} \\
& \leq\left(a_{n}^{f(n)} q_{n}^{2}(B)\right)^{-1} \leq\left(q_{n}(A)\right)^{-f(n) / 2}\left(q_{n}^{2}(B)\right)^{-1} .
\end{aligned}
$$

Thus from (1)-(4), if $P_{n} \neq 0$, we have

$$
r^{n D_{2} / 2}\left(q_{n}(A)\right)^{-D_{1}-D_{2}} \leq O\left(\left(q_{n}(A)\right)^{-f(n) / 2}\right)
$$

which is a contradiction when $n \in\left(n_{j}\right)$ is sufficiently large. To complete the proof, we need only show that $P_{n} \neq 0$ for infinitely many $n \in\left(n_{j}\right)$. First we observe that at least one of the $w_{1}, w_{2}$ is $\neq 0$. For if not, unless $P(X, Y)$ is constant with respect to one of its arguments, $A$ and $B$ would satisfy a polynomial equation of total degree lower than $D_{1}+D_{2}$, namely,

$$
\sum_{i, j} i w_{i j} X^{i-1} Y^{j}=0 \text { or } \sum_{i, j} j w_{i j} X^{i} Y^{j-1}=0 .
$$

Since it involves only minor change of arguments for the case where only one of the $w_{1}, w_{2}$ is 0 , we shall assume that none of the $w_{1}, w_{2}$ is 0 . We also observe that from parts (i) and (ii) of the lemma,

$$
\left|\frac{\delta_{1}}{\delta_{2}}\right| \leq \frac{2 q_{n}(B) q_{n+1}(B)}{q_{n}(A) q_{n+1}(A)} \leq 2\left(r^{n / 2+(n+1) / 2}\right)^{-1} \rightarrow 0 \quad\left(n \in\left(n_{j}\right), n \rightarrow \infty\right) .
$$

Consequently, $P_{n}=\delta_{2} Q$ where

$$
Q=w_{1} \delta_{1} / \delta_{2}+w_{2}+O\left(|\delta|^{2} / \delta_{2}\right) \rightarrow w_{2} \neq 0 \quad\left(n \in\left(n_{j}\right), n \rightarrow \infty\right)
$$

and so $P_{n} \neq 0$ for infinitely many $n \in\left(n_{j}\right)$. This completes the proof of the theorem. 
4. Proof of the Corollary. Let

$$
A=\sum_{i=1}^{\infty}\left(g_{1}-1\right) g_{1}^{-[i \beta]}, \quad B=\sum_{i=1}^{\infty}\left(g_{2}-1\right) g_{2}^{-[i \beta]}
$$

and let the continued fractions of $A, B, \beta^{-1}$ be

$$
\left[a_{0} ; a_{1}, a_{2}, \ldots\right],\left[b_{0} ; b_{1}, b_{2}, \ldots\right],\left[c_{0} ; c_{1}, c_{2}, \ldots\right],
$$

respectively. From Bundschuh [2, Theorem 1, p. 111], we know

$$
\begin{aligned}
& a_{n}=g_{1}^{q_{n-2}} \sum_{i=0}^{c_{n}-1} g_{1}^{i q_{n-1}}, \quad(n=1,2,3, \ldots) \\
& b_{n}=g_{2}^{q_{n-2}} \sum_{i=0}^{c_{n}-1} g_{2}^{i q_{n-2}},
\end{aligned}
$$

where $q_{n}$ denotes the denominator of the $n$th convergent of the continued fraction of $\beta^{-1}$. Since $g_{1}>g_{2}$, then

$$
\begin{aligned}
a_{n} & \geq g_{1}^{q_{n-2}} \sum_{i=0}^{c_{n}-1} g_{2}^{i q_{n-2}} \\
& =\left(g_{1} / g_{2}\right)^{q_{n-2}} g_{2}^{q_{n-2}} \sum_{i=0}^{c_{n}-1} g_{2}^{i q_{n-2}} \quad(n=1,2,3, \ldots) \\
& \geq\left(g_{1} / g_{2}\right) g_{2}^{q_{n-2}} \sum_{i=0}^{c_{n}-1} g_{2}^{i q_{n-2}}=\left(g_{1} / g_{2}\right) b_{n},
\end{aligned}
$$

and so there exists $r=g_{1} / g_{2}>1$ such that $r^{-1} a_{n} \geq b_{n}(n=1,2,3, \ldots)$. Also, from equation (10), p. 114 of Bundschuh [2], we know that

$$
\begin{array}{cc}
b_{n} \geq g_{2}^{\left(c_{n}-1\right) q_{n-1}} & (n=1,2,3, \ldots), \\
a_{n-1}<g_{1}^{c_{n-1} q_{n-2}} & (n=4,5,6, \ldots) .
\end{array}
$$

In order to complete the proof, it suffices to show that for $n \geq 4$ there exists an integer-valued function $f(n)$ with $f(n) \geq 2, f\left(n_{j}\right) \rightarrow \infty$ for some increasing subsequence $\left(n_{j}\right)$ of positive integers, such that $b_{n} \geq a_{n-1}^{f(n-1)}$. To satisfy this last inequality, it is enough from the above estimates to have

$$
f(n-1) \leq \frac{\left(c_{n}-1\right) q_{n-1} \log g_{2}}{c_{n-1} q_{n-2} \log g_{1}}=\frac{\log g_{2}}{\log g_{1}}\left(c_{n}-1\right)\left(1+\frac{q_{n-3}}{c_{n-1} q_{n-2}}\right) .
$$

Choose

$$
f(n-1)=\left[\frac{\log g_{2}}{\log g_{1}}\left(c_{n}-1\right)\right] \quad(n=4,5,6, \ldots) .
$$

From the hypotheses that $\left(c_{n}\right)$ is an unbounded sequence each of which is $\geq 1+$ $2 \log g_{1} / \log g_{2}$, we immediately infer that $f(n) \geq 2$ and $f\left(n_{j}\right) \rightarrow \infty$ for some increasing sequence of positive integers $\left(n_{j}\right)$. 


\section{REFERENCES}

1. W. W. Adams and J. L. Davison, A remarkable class of continued fractions, Proc. Amer. Math. Soc. 65 (1977), 194-198.

2. P. Bundschuh, Über eine Klasse reeller transzendenter Zahlen mit explizit angebbarer $g$-adischer und Kettenbruch-Entwicklung, J. Reine Angew. Math. 318 (1980), 110-119.

3. ___ Transcendental continued fractions, J. Number Theory 18 (1984), 91-98.

4. A. Durand, Indépendance algébrique de nombres complexes et critére de transcendance, Compositio Math. 35 (1977), 259-267.

5. Y. Flicker, Algebraic independence by a method of Mahler, J. Austral. Math. Soc. Ser. A 27 (1979), 173-188.

6. G. H. Hardy and E. M. Wright, An introduction to the theory of numbers (4th ed.), Oxford Univ. Press, London, 1971.

7. I. Shiokawa, Algebraic independence of certain gap series, Arch. Math. 38 (1982), 438-442.

Department of Mathematics, Kasetsart University, BangkoK 10900, ThaiLAND 